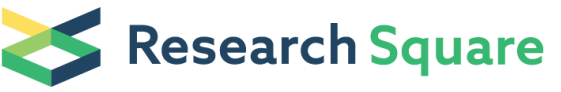 \\ Preprints are preliminary reports that have not undergone peer review. \\ They should not be considered conclusive, used to inform clinical practice, \\ or referenced by the media as validated information.
}

\section{Blockchain-based Multi-skill Mobile Crowdsourcing Services}

\section{Weize XU}

Zhejiang University School of Medicine

\section{Hongyue Duan}

Hangzhou Dianzi University School of Computer Science and Technology

Jie Huang ( $\nabla$ huangjie@zust.edu.cn )

Hangzhou Dianzi University School of Computer Science and Technology https://orcid.org/0000-0001-

9717-8355

\section{Deyong Liu}

Hangzhou Dianzi University School of Computer Science and Technology

\section{Yichao Chen}

Hangzhou Dianzi University School of Computer Science and Technology

\section{Research}

Keywords: Mobile crowdsourcing, Blockchain, Spatial communication, Smart Contract

Posted Date: January 24th, 2022

DOI: https://doi.org/10.21203/rs.3.rs-1272507/v1

License: (c) (i) This work is licensed under a Creative Commons Attribution 4.0 International License.

Read Full License 


\title{
RESEARCH
}

\section{Blockchain-based Multi-skill Mobile Crowdsourcing Services}

\author{
Weize $\mathrm{Xu}^{1}$, Hongyue Duan ${ }^{2}$, Jie Huang ${ }^{3 *}$, Deyong $\mathrm{Liu}^{2}$ and Yichao Chen ${ }^{2}$
}

\author{
${ }^{*}$ Correspondence: \\ huangjie@zust.edu.cn \\ ${ }^{3}$ School of Information and \\ Electronic Engineering, Zhejiang \\ University of Science and \\ Technology, Hangzhou, China \\ Full list of author information is \\ available at the end of the article
}

\begin{abstract}
With the boom in $5 \mathrm{G}$ technology, mobile spatial crowdsourcing has shown great dynamism in industrial mobile communications and edge computing node management. But the traditional crowdsourcing system is not enough to adapt to the new environment. In traditional crowdsourcing, the crowdsourcing process is usually managed through a centralized crowdsourcing platform. The centralized crowdsourcing platform faces the following problems: 1) Single point of failure. 2) User privacy leakage. 3) Subjective arbitration. 4) Additional service fee. 5) The task assignment process is not transparent. To solve those problems, this paper uses a decentralized blockchain to replace the centralized crowdsourcing platform, thereby realizing a decentralized crowdsourcing system. And we analyzed the problems faced by multi-skilled spatial crowdsourcing tasks in the blockchain crowdsourcing system. In addition, a crowdsourcing task allocation algorithm is proposed, which realized the transparent task distribution process and adapts to the computing-constrained environment on the blockchain. Compared with the TSWCrowd blockchain-based crowdsourcing model, our system has a higher task allocation rate under the same conditions. And the experimental result shows our system has good economic feasibility, which decentralizes the crowdsourcing process and significantly reduces the additional consumption of the crowd-sourcing process.
\end{abstract}

Keywords: Mobile crowdsourcing; Blockchain; Spatial communication; Smart Contract

\section{Introduction}

With the boom in 5G technology, mobile spatial crowdsourcing has shown great dynamism. When crowdsourcing meets blockchain, a wonderful fusion occurs between the decentralized idea of blockchain and the idea of crowdsourcing to solve problems by relying on the wisdom of the crowd. The blockchain + crowdsourcing model is useful in people's daily life, industrial mobile communications, and edge computing node management. Many excellent features of blockchain can be used to solve problems related to dynamic configuration updates, network security, and load balancing in industrial mobile communications. The industrial mobile system can build the infrastructure through the blockchain, and then use the crowdsourcing mechanism to solve the business and application problems of the system.

Crowdsourcing, starting from online simple crowdsourcing tasks, which can be easily accomplished by workers online, such as data annotation and T-shirt design. Developing into complex spatial crowdsourcing tasks, which requires workers to physically move to some specified locations and accomplish these tasks. 
[1] proposed a new complex crowdsourcing task model: Multi-Skill required spatial crowdsourcing task, which is a model closer to real life. For example, a requester wants to hold a party, and he needs to hire some workers to help him cook, play music, and take photos. This task is a typical multi-skill crowdsourcing task, which requires the participating workers to have matching skills, such as playing instruments, cooking, and photography. Especially with the development of GPSequipped smart devices and wireless mobile networks. Spatial crowdsourcing tasks are gradually commercialized and become a very successful business model in recent years. Platforms such as MTurk[2], Uber[3], TaskRabbit[4] are developing greatly rapidly.

In general, a crowdsourcing system consists of three groups of roles: requesters, workers, and a centralized crowdsourcing platform (Fig. 1). As shown in the figure, the centralized crowdsourcing platform controls all the crowdsourcing processes. However, this kind of crowdsourcing system based on a centralized platform has many shortcomings. Disadvantages of the centralized crowdsourcing platform listed in [5]: 1) Single point of failure, which generally occurs in centralized systems. 2) User privacy leakage. User's privacy information(e.g., name, email address, and phone number) are saved in the database of crowdsourcing platforms, which has the risk of privacy leakage and data loss. 3) Subjective arbitration. When there is a disagreement between the requester and the worker, they will get help from the crowdsourcing platform to give a subjective arbitration, which may lead to "false-reporting" and "free-riding" [6]. 4) Additional service fee. As usual, when a crowdsourcing task is finished, the crowdsourcing platform will require users to pay for services, which increases users' costs.

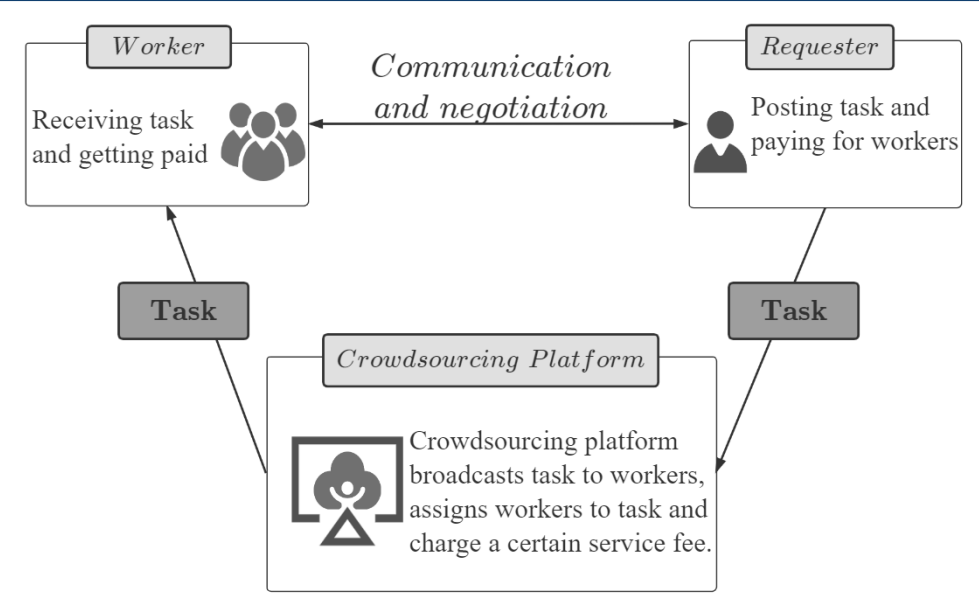

Figure 1 Crowdsourcing System

In order to solve the centralized platform problems, many researchers have introduced blockchain technology into the crowdsourcing process and replaced the traditional centralized crowdsourcing platform with blockchain, a decentralized infrastructure. Yuan et al.[7] is the first to design and implement a private and anonymous blockchain-based crowdsourcing system, and solve the problem of data leakage and identity leakage in a decentralized crowdsourcing system. [5] designed a decentralized crowdsourcing system with reliability, fairness, security, and low services 
fee. Liu et al.[8] proposed a system for blockchain-based software crowdsourcing. Yuan et al. [9] using a blockchain-based crowdsourcing system to outsource computationally intensive tasks in machine learning.

However, the current blockchain-based crowdsourcing system usually sets the goal to design and implement a general decentralized crowdsourcing framework. Seldom of they pay attention to the problem of task assignment. They usually use an intuitive method for task assignment, which is a first-come, first-serve mechanism. At the same time, the crowdsourcing tasks they have involved are usually simple online crowdsourcing tasks. If a qualified worker first claims a task, then assign the task to the first-claim worker. Nowadays, people's demand for spatial crowdsourcing tasks with multi-skill requirements is increasing, and such a system may lack potential.

Thus, this research is motivated by this: can we design a blockchain-based crowdsourcing system that supports spatial crowdsourcing tasks with multi-skill requirements, while having reliability, fairness, security, and a transparent task assignment mechanism. We can ensure that the execution process of the task assignment algorithm is credible and transparent by using smart contract technology. However, since each node in the blockchain network stores a complete copy of the transaction, when the number of tasks and workers to be allocated in the system is too large, the gas fee generated by the task assignment algorithm executed on-chain may bring additional costs and worse the gas fee may exceed the gas limitation. The main challenge is: how to design a fair and transparent task assignment algorithm for a crowdsourcing system to adapt to the blockchain environment with limited computing resources. And to meet the demand: spatial crowdsourcing tasks with multiple skills requirements. The proposed task assignment algorithm should take into account many factors such as spatial coordinates, skill requirements, and budget constraints. To solve the above problems, we have implemented a task assignment algorithm and a decentralized crowdsourcing system. Highlights of our original contributions in this paper are as follows.

1) We discussed the decentralized workflow of the blockchain crowdsourcing system and analyzed the problems faced by multi-skilled spatial crowdsourcing tasks in the blockchain crowdsourcing system. And we have built a crowdsourcing system based on blockchain technology, using smart contracts to realize the interaction between requesters and workers, ensuring that the crowdsourcing system is completely decentralized and fair.

2) We deployed our task assignment algorithm with smart contracts and ensured the transparency and fairness of the algorithm execution process. Finally, we implemented a crowdsourcing-based task quality evaluation mechanism through the oracle contract to ensure the fairness of crowdsourcing participants.

3) We make comparable experiments to compare with TSWCrowd. Experimental results show that our system achieves reliable task matching with lower cost and higher task allocation rates.

The remainder of the paper is organized as follows. The second part shows the related work and the third part discussed the preliminary and problem statement. The Fourth part describes the proposed system architecture. The fifth part analyzed the feasibility of the system. The final part carries on the experiment and makes the result analysis, and the fifth part summarizes the whole article. 


\section{Related Works}

In this section, we will describe the proposed task allocation strategy. There are three related topics in our research: spatial crowdsourcing task matching, smart contract on Ethereum, and the blockchain-based crowdsourcing system.

\subsection{Spatial Crowdsourcing Task Matching}

Spatial Crowdsourcing platform[10] requires workers to physically move to a certain location, and complete the crowdsourcing task, such as taking photos, cleaning a room, and repairing a computer.

According to the task allocation mechanism, spatial crowdsourcing can be also classified into two categories: 1) WST(worker selects task), with the help of a recommendation algorithm[11][12], the platform pushes tasks to workers who may be interested in. 2) TSW(task selects worker), the platform allocates tasks to workers with optimization goals such as maximum profit. In order to maximize the task allocation rate, the TSW mechanism is used in our system.

Tong et al. [13] proved that the greedy algorithm performs well for some online task matching problems. But they mainly study the model which is one worker finishes one task. In our system, the spatial task requires multiple skills. In other words, tasks in our system may need multiple workers to complete. Song et.al [14] study the multi-skilled crowdsourcing task assignment problem in real-time spatial crowdsourcing. They believe that the workers and tasks appear dynamically and task assignments should be performed in real-time. But the proposed online-exact algorithm's high complexity will limit its application in the blockchain-based crowdsourcing system. Our system also used the online task allocation mechanism. When a new task is added, the system will assign the optimal team of workers.

Peng et al. [1] researched the multi-skilled spatial crowdsourcing problem. They want to find an optimal worker-and-task assignment strategy. And they proposed three effective methods to allocate workers to tasks, including greedy, g-divideand-conquer, and cost-model-based adaptive algorithms. In this paper, the problem of multi-skilled spatial crowdsourcing is defined, and they proved that the problem is NP-hard. But the algorithm complexity of this work is not suitable for the blockchain environment.

The current crowdsourcing task allocation mechanism relies on the operation and maintenance of a centralized crowdsourcing platform. Therefore, the opacity of the task allocation process is a shortcoming of the centralized crowdsourcing system. There is no guarantee that the platform will perform a dishonest assignment for its benefit. To build a transparent task allocation process, we will introduce the blockchain-based crowdsourcing system to guarantee users' benefit.

\subsection{Smart Contract on Ethereum}

IBM[15] gives a simple definition of smart contract on blockchain: Smart contracts are simply programs stored on a blockchain that run when predetermined conditions are met. They typically are used to automate the execution of an agreement so that all participants can be immediately certain of the outcome, without any intermediary's involvement or time loss. They can also automate a workflow, triggering the next action when conditions are met. 
Since smart contracts are usually deployed on the blockchain, they have some unique characteristics. First, the program code of the smart contract will be recorded and verified by the blockchain nodes. Therefore, once the contract is deployed, it cannot be modified. Second, smart contracts are executed between anonymous and trustless independent nodes, without centralized control and coordination with third-party authorities, which can ensure transparent and fair execution. Third, the smart contract can have its digital encryption currency or other digital assets, and complete the transfer of assets when the predefined conditions are triggered[16].

Ethereum uses the Ethereum Virtual Machine (EVM) to implement a public blockchain platform that supports Turing-complete smart contracts. The entire Ethereum system can be regarded as a singleton distributed state machine. Each node in the Ethereum network runs an EVM instance and uses a consensus mechanism to ensure that the local EVM has the same state as the EVM of other nodes. Many high-level programming languages can be used to write Ethereum smart contracts, such as Solidity and Serpent, and the contract code is compiled into EVM bytecode and deployed on the blockchain for execution.

Ethereum is currently the most popular smart contract development platform and can be used to design various decentralized blockchain applications (DApps), such as digital rights management, data sharing, and crowdfunding[17].

\subsection{Blockchain-based crowdsourcing system}

With the development of blockchain technology, blockchain 2.0 technology is proposed by the Ethereum Blockchain Platform. It supports the Turing complete smart contracts. To improve the crowdsourcing process by taking advantage of the various features of blockchain technology, many researchers use smart contract technology to deploy crowdsourcing systems to the blockchain platform.

M. Li et al. [5] proposed a general decentralized crowdsourcing system framework based on blockchain, which is named CrowdBC. CrowdBC uses smart contract technology to deploy a crowdsourcing system on the blockchain. According to the classification of task allocation mechanism mentioned above, CrowdBC is a WST system. But recommendation algorithm is difficult to execute on-chain, because of the limited computing resources. Therefore, the system may face the problem of a low task allocation rate.

Maha Kadadha et al. [18] proposed to run the crowdsourcing platform entirely on Ethereum blockchain while incorporating auctions. They used a Repeated-SingleMinded Bidder (R-SMB) auction mechanism to assign workers to the task. This work introduced a task allocation mechanism for the blockchain-based crowdsourcing system. Among the many workers bidding for a task, the best one is selected and assigned the task. The system is more suitable for scenarios with fewer tasks and more workers, but the problem of low task allocation rate has not been resolved.

Liping Gao et al. [19] proposed a blockchain-based crowdsourcing framework TSWCrowd (Task Select Worker Crowd). In this framework, tasks are sorted according to specific rules, thus tasks with higher priority are assigned to workers earlier. The task allocation mechanism only considers the time and distance priority and is not suitable for spatial crowdsourcing tasks with multi-skill requirements. In the following section, we implemented a FIFO algorithm to simulate the allocation mechanism of the TSWCrowd system. 
In summary, no system can not only guarantee the rationality of the task allocation scheme in the blockchain environment and at the same time consider the spatial crowdsourcing task with multi-skill requirements.

\section{Methods}

\subsection{Preliminary And Problem Statement}

Blockchain-based crowdsourcing system is no longer a new vocabulary. Many works have proved the feasibility and security of blockchain-based crowdsourcing. However, most of the previous work is aimed at simple crowdsourcing scenarios, and this article is dedicated to extending the blockchain crowdsourcing system to a more complex crowdsourcing scenario: multi-skilled spatial crowdsourcing and its task allocation process.

In this section, we analyze the decentralized workflow of the Ethereum blockchain to verify the feasibility of our work and explore the challenging issues that may be faced. Ethereum blockchain is a decentralized platform, and its security is guaranteed by a total of 2,722 miner nodes[20] in the global Ethereum network.

\subsubsection{Decentralized Workflow of Blockchain}

As Fig.2 shows, we can think of Ethereum as a distributed singleton database, and each miner node is a complete backup of the database. Talk to a database with more than 2,700 backups, the data writing operations need to be very cautious. In programming, the usual practice is to lock the database when data needs to be updated to ensure that only one person(Primary) can write to the database at the same time to avoid data consistency-related problems. Ethereum blockchain uses the proof-of-work mechanism to guarantee that only one person can write to the Ethereum state database at a time. Proof-of-work is a competitive consensus algorithm. Nodes with stronger HashRate have a greater probability of winning the competition. This article does not elaborate on the consensus mechanism of proof-of-work. In short, the mechanism will select a node to update the Ethereum database every $13 \mathrm{~s}$.

The release of a new block represents an update of the Ethereum state database, and each block contains many transactions. These transactions include transfer transactions, smart contract deployment transactions, and smart contract invocation transactions. After the new block is released, other miner nodes serve as backups of the database and need to update their local database to be consistent with the primary database. Therefore, the other miner nodes will verify the newly released block and execute the transfer, contract deployment, and contract invocation operations with the time order in the new block. Ethereum guarantees the consistency of the results of operations, especially for contract calls, the same input will produce the same output. In this way, the Ethereum system has completed a round of data updates, which means that part of the transactions submitted by users is executed by the Ethereum system. Every transaction has been verified and executed by all the honest nodes in the global Ethereum network. As long as the HashRate of honest nodes accounts for more than $51 \%$ of the network, then Ethereum can be considered sufficiently secure. There have been many works that have analyzed and discussed the security of the Ethereum system. We can think that once a transaction is uploaded into the blockchain, the consequences of the transaction are irreversible. 


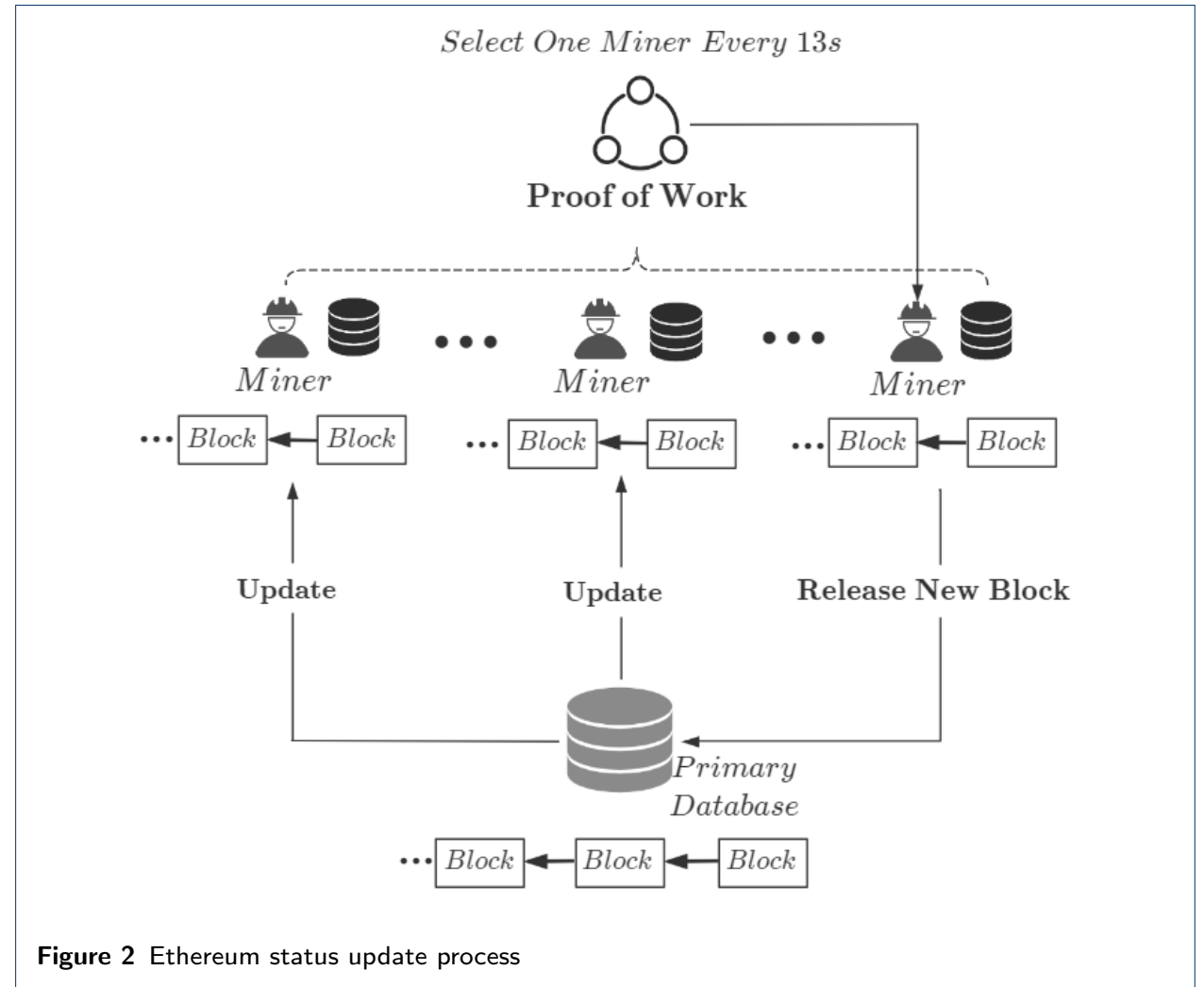

\subsubsection{Problem Statement}

We described the decentralized workflow of the Ethereum blockchain, and the decentralized crowdsourcing system based on the blockchain also operates in the same process. CrowdBC represents the current blockchain crowdsourcing system. In simple terms, CrowdBC is more like a public whiteboard in the community. Requesters can post tasks on the whiteboard, and workers can accept tasks on the whiteboard. Although CrowdBC also involves the historical reputation evaluation of workers and the minimum requirements of tasks and other features. It does not involve any task assignment process. Workers and tasks are matched according to personal wishes, which may lead to a low task assignment rate. For this reason, we have included a multi-skilled spatial crowdsourcing task allocation algorithm in our system, and we are committed to implementing this allocation algorithm through an open and transparent Ethereum smart contract. The code of the algorithm is publicly deployed on Ethereum, and the process of algorithm execution is also verified by more than 2,700 miner nodes on Ethereum. But this also brings the problem about the time and space complexity of the algorithm. Every calculation and storage in the algorithm is executed in all miner nodes. To avoid wasting resources and reward miners, Ethereum stipulates that miners can charge for these calculations and storage processes. Therefore, we propose the first challenge: 1) Make the final task allocation rate of the task allocation algorithm as high as possible while limiting the time and space complexity of the algorithm.

In the Ethereum system, all operations are processed in the form of transactions. Ethereum transactions have a certain delay problem, which is mainly due to the 
13 s block time set by the Ethereum system. For spatial crowdsourcing, time is a very important constraint. If a task released by the requester needs to be completed within 3 hours, but the task release process takes 1 hour, it must be an unacceptable time delay. Therefore, the second challenge is: 2) Whether the average transaction latency of Ethereum can support multi-skill spatial crowdsourcing tasks. In fact, the transaction delay of Ethereum can be controlled, and this issue will be analyzed in detail later.

In the blockchain crowdsourcing system, a decentralized blockchain platform replaces the traditional centralized crowdsourcing platform. Without the supervision of a trusted third party, whether the trust relationship established by the requester and the worker through the blockchain is reliable. The general steps of the blockchain crowdsourcing system is: 1) The requester publishes tasks through smart contracts. 2) Workers receive tasks through smart contracts. 3) Workers complete the task and get the reward. However, both workers and requesters have incentives to profit by not following the process. For example, workers may falsely report that they have completed tasks to obtain task rewards. The requester may deliberately evaluate the workers' work as low quality, thereby reducing their expenses. In CrowdBC, a solutionEvaluate $(\cdot)$ function is used to evaluate the quality of the task. This function is set up when the task is released, and the quality of crowdsourcing tasks is evaluated through code logic. This method may be effective for some simple online crowdsourcing tasks (e.g. data labeling, image recognition, etc.), but it may not be feasible for multi-skilled spatial crowdsourcing. For example, the requester has issued a task that needs to repair the water pipe. It is difficult to say that the pre-set code logic can be used to determine whether the water pipe is repaired or not. So far we can put forward the third challenge: 3) In the decentralized blockchain platform, how to implement an effective task quality evaluation mechanism for multi-skilled spatial crowdsourcing tasks.

\subsection{System Architecture}

\subsubsection{Overview}

After the previous analysis, our proposed blockchain crowdsourcing system needs to implement the following modules.

1) Decentralized crowdsourcing process. For example, the basic processes of crowdsourcing such as user registration, post task, and reward distribution. To decentralize the crowdsourcing processes, we designed two smart contracts to manage the crowdsourcing process, namely Worker Manager Contract and Task Manager Contract.

2) Efficient task allocation algorithm. The multi-skilled spatial crowdsourcing task allocation is a complicated problem. Many works have discussed this problem. Because of the limited computing and storage of the blockchain, these methods in previous works are difficult to apply to the blockchain crowdsourcing system. To reduce the gas consumption of the algorithm and improve the task allocation rate, we designed a task allocation algorithm with a greedy strategy. The algorithm is deployed in the Task Manager Contract to ensure the openness and transparency of the task assignment process.

3) Reasonable and effective task acceptance mechanism to ensure the fairness of the crowdsourcing process. While the blockchain is decentralized, 
it also brings the problem of weak supervision. After losing the supervision of the centralized crowdsourcing platform, crowdsourcing participants may deceive others to profit for themselves. Therefore, we designed and implemented a crowdsourcingbased task quality evaluation mechanism to ensure the interests of crowdsourcing participants through a decentralized supervision method.

In this section, we first explain the concrete implementation of our system from the above three aspects and then introduce the crowdsourcing process.

\subsubsection{Implementation Of The Decentralized Crowdsourcing Process}

The proposed blockchain-based crowdsourcing system is built by smart contracts. We implement two types of smart contracts: Worker Manager Contract(WMC), Task Manager Contract(TMC). Through these contracts and methods, we deploy the crowdsourcing process on the decentralized Ethereum blockchain.

\section{Worker Manager Contract}

In multi-skill spatial crowdsourcing scenario, the worker is a complex entity, which is called a multi-skilled worker. Multi-skilled workers' data structure is shown in the figure.

Worker $P$ is a set of multi-skilled workers. For a worker, Latitude and Longitude represent the specific location in timestamp $T$, Skills represents the skills that the worker has mastered, and $D$ represents the maximum distance of the worker willing to move. Address represents the worker's Ethereum account address.

The main functions of this contract are shown in the figure. WorkerRegister $(\cdot)$ is used to initialize a new worker and store the worker's information in the WorkerP set. Workers can change their real-time location through the SetLocation(·) function, and other information( Skill and $D$ ) through SetInfo(.) function.

\begin{tabular}{|c|c|c|}
\hline \multicolumn{3}{|c|}{ Worker Manager Contract } \\
\hline \multicolumn{3}{|c|}{ Multi-Skilled Worker : } \\
\hline Longitud(uint) & Latitude(uint) & Skills(uint[]) \\
\hline $\mathrm{T}$ (uint) & $\mathrm{D}$ (int) & Address \\
\hline A Set of Multi-Skil & ed Worker : & WorkerP[ ] \\
\hline Function & Parameters & Return \\
\hline WorkerRegister( ) & Worker & bool \\
\hline SetLocation( ) & Longitud, Latitude & bool \\
\hline SetInfo( ) & Skill、D & bool \\
\hline
\end{tabular}

Figure 3 Worker Manager Contract

\section{Task Manager Contract}

The multi-skilled task's data structure is described in Fig.4, TaskP is a set of multiskilled tasks. Latitude and Longitude represent the specific location. $T$ is the task's start time and dur is the duration time. So we can calculate that the deadline of a task is $T+$ Dur, and workers are required to finish the tasks before the deadline. Skills represents the skill requirements by a task. Furthermore, each task is 
associated with a budget, $B$, which is the salaries for workers. Rquester Address address represents the requester's Ethereum account address. Assignment [task]team represents the mapping between a task and a team, indicating the assignment relationship.

The main functions of this contract are shown in the figure. The requester can create a new crowdsourcing task by PostTask $(\cdot)$ function. When the PostTask function is called, the requester has to deposit some eth in the contract as mortgage payments to prevent the "free-riding" attack. The mortgage payments amount is required to be no less than the budget. When the deposited mortgage payments are confirmed, the contract will store the newly created task in TaskP. After the task is successfully released, the requester can call the TaskAssign $(\cdot)$ function. The TaskAssign $(\cdot)$ function will select a team from all the assignable workers for this task. The assignment instance returned by the function will be recorded in map Assignment [task]team. The task allocation algorithm will be explained in detail in the next section.

After the task is completed by works, the Payment $(\cdot)$ function will pay the workers according to the evaluation score of the task. The task quality evaluation mechanism will be introduced in the later section. The requester can retrieve the remaining budget through the withdraw(.) function. Or if the task is not completed after the deadline, the requester can retrieve all the budget using the withdraw $(\cdot)$ function.

Task Manager Contract

\begin{tabular}{|c|c|c|}
\hline \multicolumn{3}{|c|}{ Multi-Skilled Task : } \\
\hline Longitud(uint) & Latitude(uint) & Skills(uint[]) \\
\hline $\mathrm{T}$ (uint) & Dur(uint) & RequesterAddress \\
\hline \multicolumn{2}{|c|}{ A Set of Multi-Skilled Task : } & TaskP[ ] \\
\hline \multicolumn{3}{|c|}{ A Map of Assignment Instance: } \\
\hline Function & Parameters & Return \\
\hline PostTask( ) & Task & bool \\
\hline Withdraw( ) & Message.sender & bool \\
\hline TaskAssign( ) & Task, WorkerP[] & Assignment Instance \\
\hline Payment( ) & Team [], Task, Score & bool \\
\hline
\end{tabular}

Figure 4 Task Manager Contract

\subsubsection{Implementation Of The Task Allocation Algorithm}

Ethereum will charge a certain gas fee for any operation that consumes storage and calculation resources. And Ethereum sets a gas limit to prevent endless loops in smart contracts, which may cause Ethereum to crash. To achieve a transparent and efficient task allocation mechanism, we deploy the proposed algorithm on Ethereum in the form of a smart contract.

\section{Task allocation algorithm}

The task allocation algorithm will consume a certain amount of gas each time 


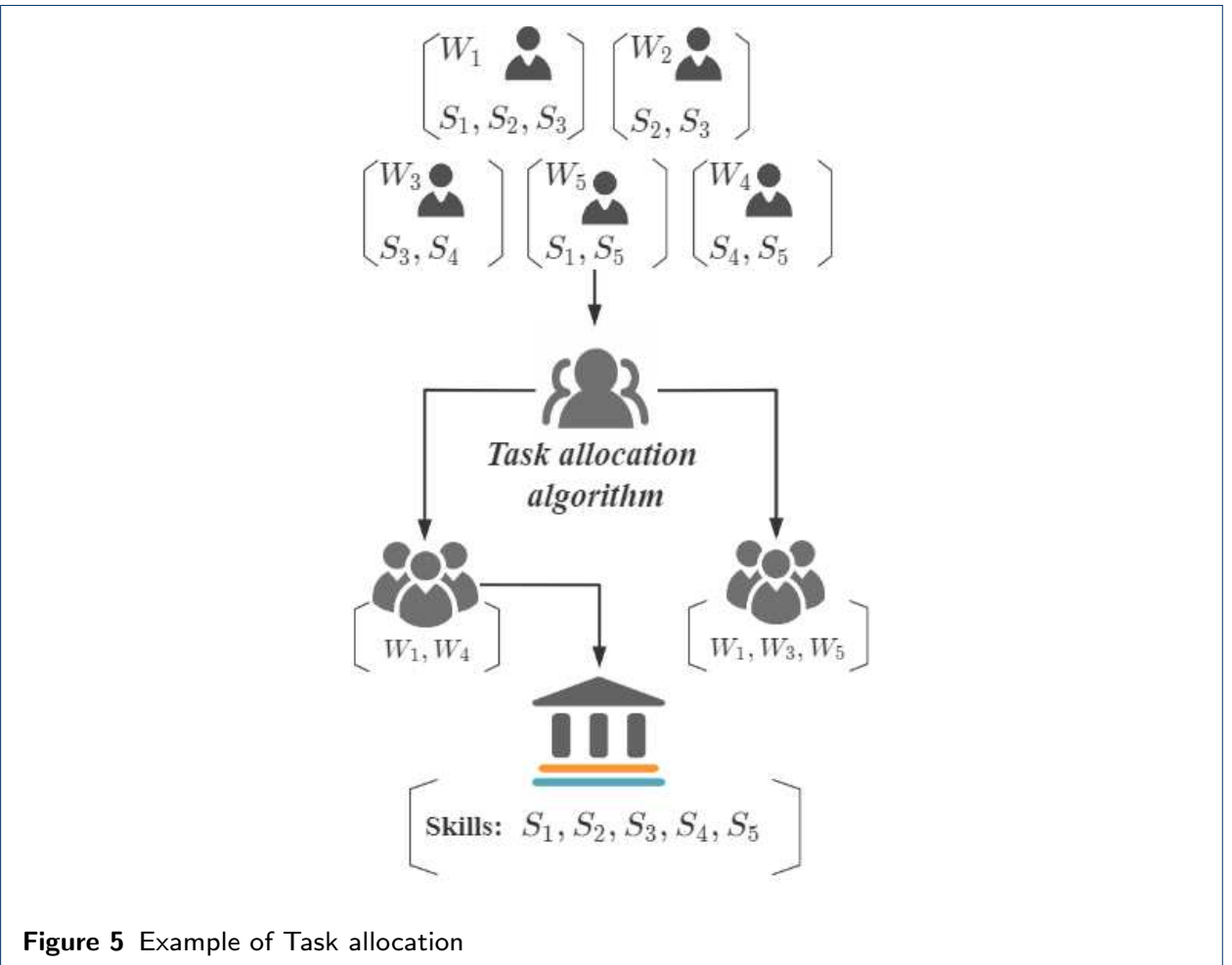

Figure 5 Example of Task allocation

it is executed. To reduce this consumption, we consider an optimization scheme for skill coverage rate. As the Fig.5 shows, there is a task requires a set of skill $\left\{S_{1}, S_{2}, S_{3}, S_{4}, S_{5}\right\}$, and a set of workers $\left\{W_{1}, W_{2}, W_{3}, W_{4}, W_{5}\right\}$. As the figure shows, every worker has mastered certain skills. In this case, two teams $\left\{W_{1}, W_{4}\right\}$ and $\left\{W_{1} W_{3}, W_{5}\right\}$ can satisfy the task's require. Under the optimization scheme for skill coverage rate, the team $\left\{W_{1}, W_{4}\right\}$ will be assigned to the task. While reducing the number of dispatched workers, it can also increase the task allocation rate. With the same set of workers and task sets, our algorithm allows more tasks to be allocated.

The definition of skill coverage rate: the size of the intersection of the worker's skills and the task's skills. As in the example above, $W_{1}$ 's skill coverage rate is 3 , $W_{2}$ 's skill coverage rate is 2 .

Algorithm1 shows the greedy framework, the output of algorithm1 is an assignment instance. An assignment instance contains a task and a set of workers, which means that these workers are assigned to the task. The function getValidWorker in the line 1 shows in the algorithm2. In this function, we find all the valid workers for a certain task from the WorkerP. And then, we use the function formTeam to group a team from the valid workers (line 2). The function formTeam shows in the algorithm3, it will form a team for the input task with a greedy strategy(lines3). Then we check whether the workers in the team can meet the skill requirements of the task and whether the budget of the task is sufficient.

In algorithm2, we select a set of workers for a task. First, we check whether there is an overlap between the skills mastered by the worker and the skills required for the task. Then we confirm that the distance between the worker and the task is less 


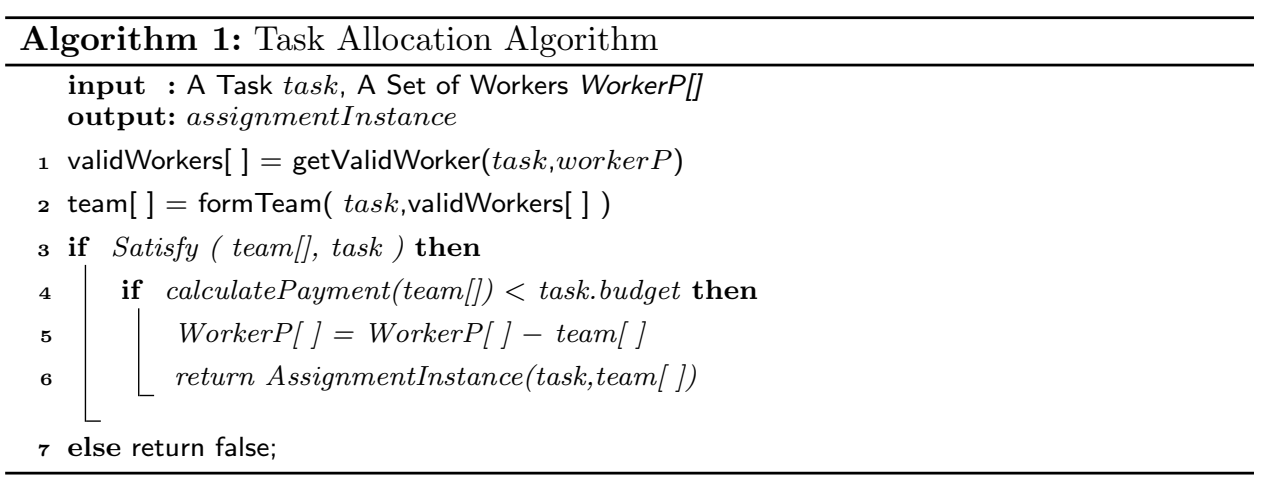

than the Workeri.d (the maximum distance of the worker willing to move). In this way, we can ensure that the selected workers are effective for this task.

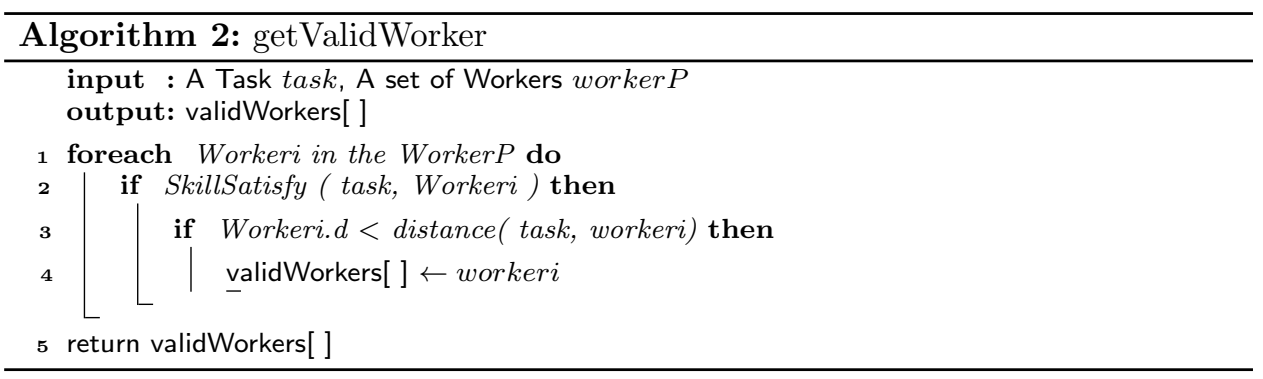

Algorithm3 shows the formTeam function. In each loop, we select a worker from validWorkers[] with a maximum skill coverage rate. Due to Ethereum's gas limit, we need to set a maximum number of loop executions to prevent endless loops.

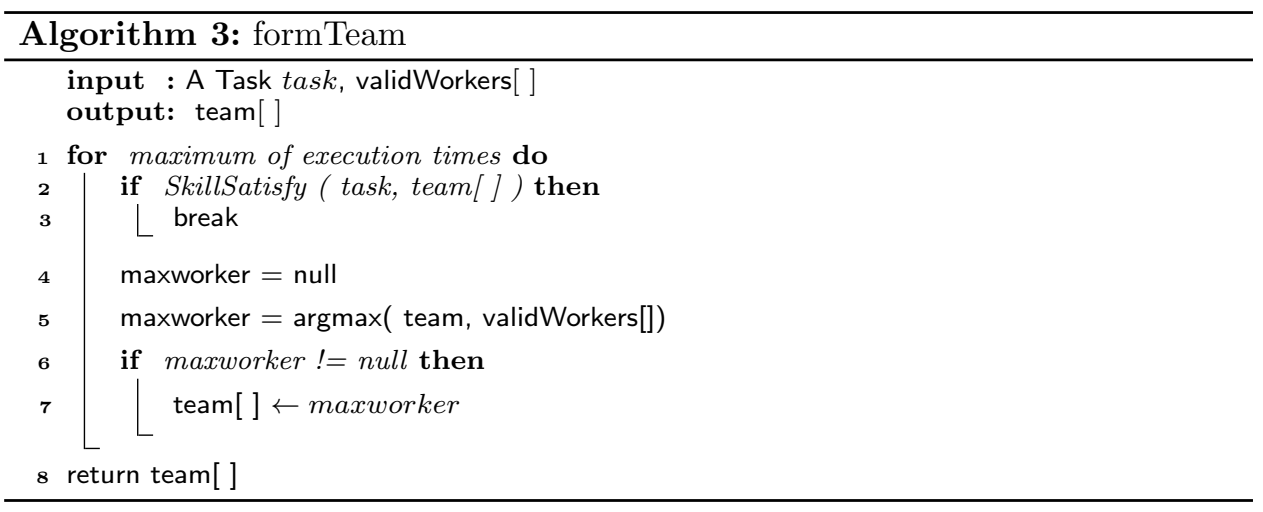

The Algorithm4 argmax can find the maxWorker with the highest skill coverage rate from all the valid workers. First, we calculate the current team[]'s skill coverage rate(lines2). And then we traverse all workers in validWorkers[] to find the maxWorker.

\section{FIFO task allocation algorithm}

In addition to the greedy algorithm, we also implemented an intuitive FIFO task allocation algorithm, which allocates tasks to workers in the order in which they 


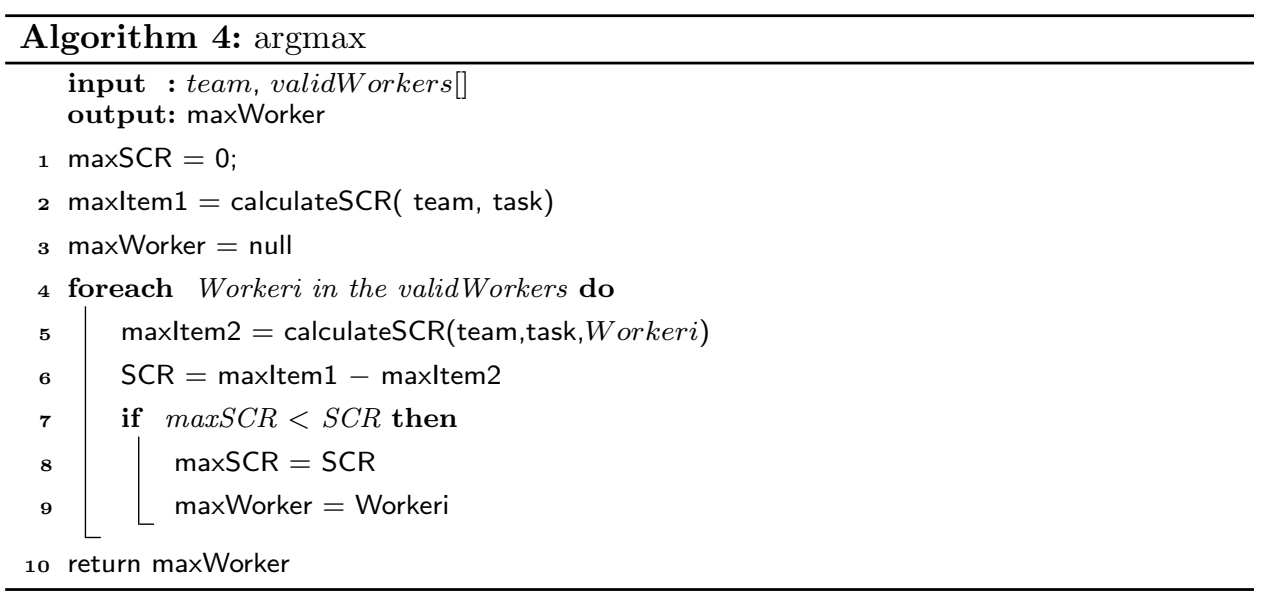

join the system. This algorithm is used as a baseline algorithm to compare our greedy algorithm. The comparison results are shown in the experimental section.

Algorithm5 shows the FIFO task allocation algorithm. The algorithm adds workers who meet the requirements to the team in chronological order until the team satisfies the task's requirements or traverses all valid workers(lines 3-8). The end of the algorithm is the same as algorithm1(lines 9-13). The algorithm does not consider the worker's task coverage rate and assigns workers to tasks with an intuitive idea, which is first-come, first-serve.

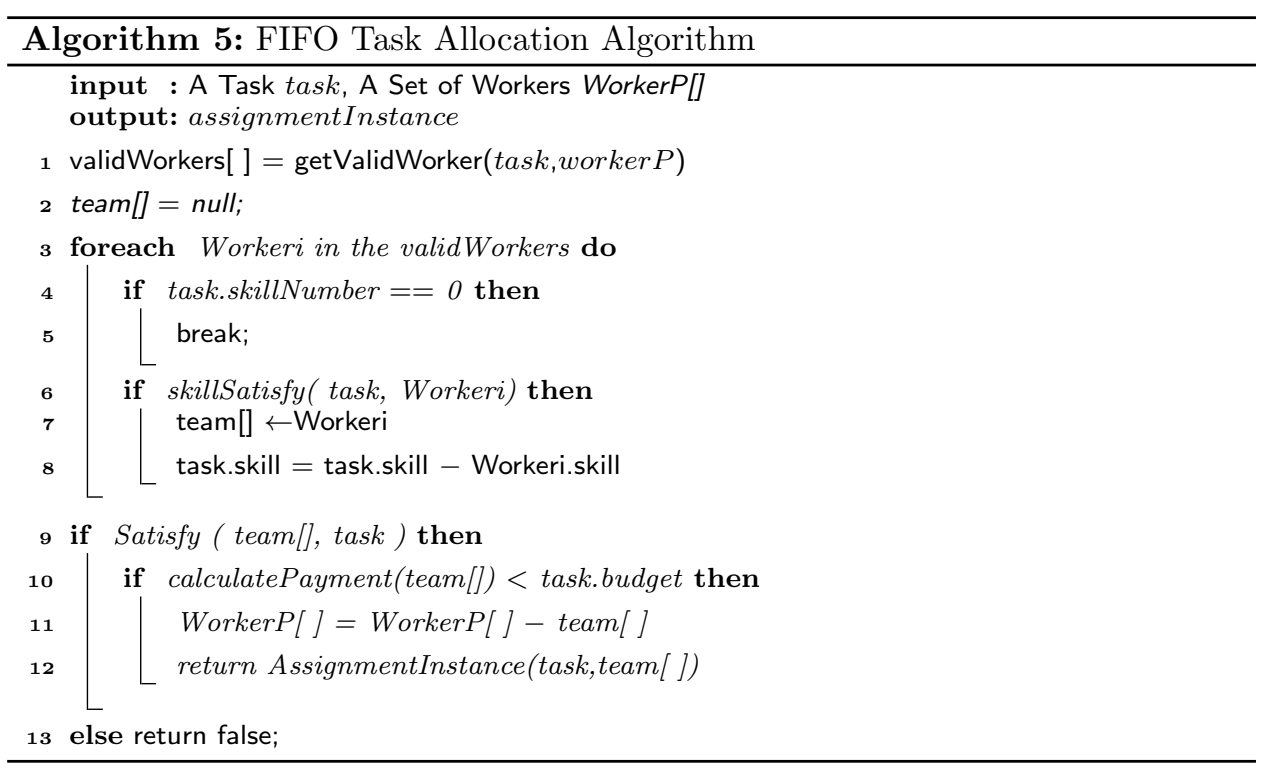

\subsubsection{The Task Quality Evaluation Scheme Based On Crowdsourcing}

In traditional crowdsourcing, the quality evaluation of tasks is supervised by the crowdsourcing platform. Through a trusted third party to solve the "false-reporting" and "free-riding" problems that may arise between the requesters and the workers. And it also brings the problem of "subjective arbitration", and fairness is difficult 


\section{Oracle Contract}

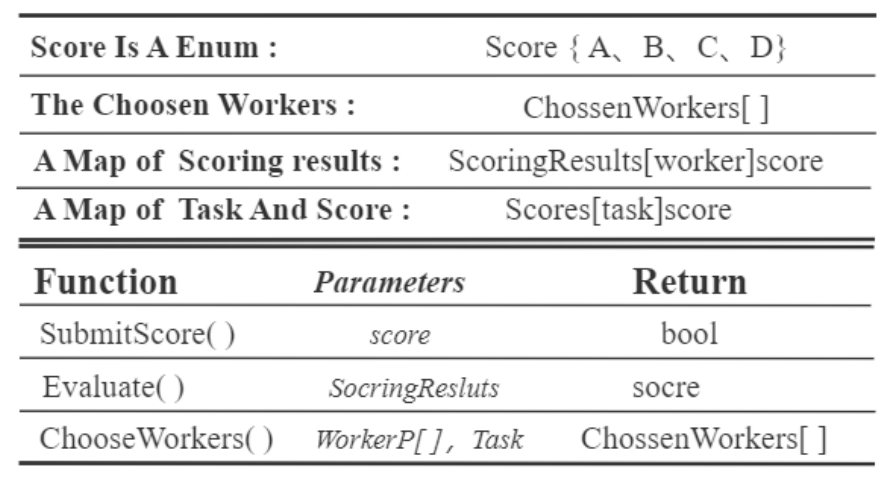

Figure 6 Oracle Contract

to guarantee. In decentralized crowdsourcing, a trusted third party is removed. But the "false-reporting" and "free-riding" issues have not been resolved. How to ensure fairness among crowdsourcing participants is the main topic of this section.

We propose a task quality evaluation scheme for spatial crowdsourcing tasks with multi-skill requirements. Because of the skill requirements, these crowdsourcing tasks are usually complicated, and it is hard for the requester to predict how the tasks will be completed. In CrowdBC, the requester announces the evaluation strategy of the task at the same time as the task is published. As stated in section 3.2, this kind of scheme is difficult to work in our scenario. Therefore, we propose a task quality evaluation scheme based on a blockchain oracle in a crowdsourcing way.

A blockchain oracle is a service that connects smart contracts with the outside world, primarily to feed information in from the world[21]. In general, we will select 10 juries to score the completion of a crowdsourcing task, then the scoring result of the jury is used as the score parameter of the Payment $(\cdot)$ function in TMC through the blockchain oracle. We implemented the oracle with a smart contract, and it shows in the figure.

Fig.7 shows the task quality evaluation process. In the first step, the contract uses the ChooseWorkers(.) function to select 10 workers as the jury. These selected workers are required to have skills that match the task. If a sufficient number of workers cannot be selected, the function will randomly select workers until the number is sufficient. Secondly, the selected workers will receive a notification and be asked to score the task based on the materials(e.g. photos of the task scenes) provided by the original participants of the task. By using the SubmitScore (·) function, those selected workers can upload their scores to the blockchain. And the SubmitScore(·) function will check whether the person who submitted the score is included in the ChossenWorkers[] set. Finally, when the contract has collected enough submissions, it will call the Evaluate $(\cdot)$ function to calculate the final result. To encourage the jury to give correct evaluations, workers who submitted the same score as the final score will be rewarded. The final score will be store in the Scores $[$ task $]$ map for the payment(·) function to query. 


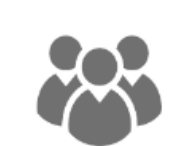

Workers

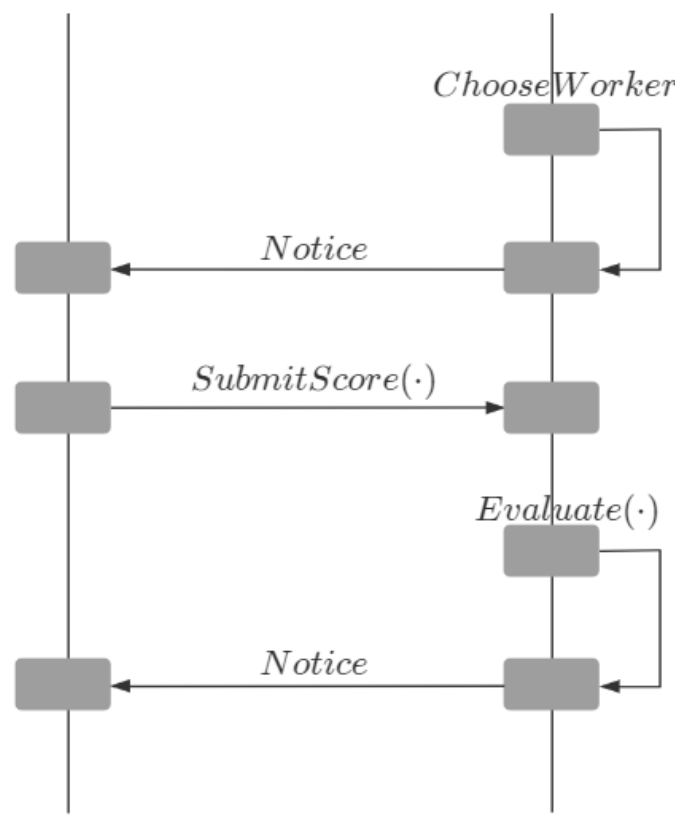

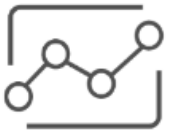

Oracle Contract $s(\cdot)$

Figure 7 Task Quality Evaluation Process

\subsubsection{System Process}

\section{User Registration}

Requesters and workers need to register in the system before they participate in the crowdsourcing process(e.g., post task, accept tasks, get payment, etc.). The Ethereum address can uniquely identify a user, workers in our system have much other information(e.g., location information, effective active time, and skills mastered) besides the identity. Therefore, workers should register other information into the system through the Worker Manager Contract.

\section{Creating task}

After the requester finished the registration, they can publish tasks through Task Manager Contract. To publish a task, requesters call the PostTask(·) function and pass in the longitude, latitude, skill requirements, and other information of the task. Furthermore, the requester should transfer the budget to the Task Manager Contract. When the task is finished, the pre-transferred budget will be used to pay the workers, and the rest will be returned to the requester.

\section{Task Assignment}

After a task is published, the requester can call the TaskAssign $(\cdot)$ function to assign workers to the published task. This function uses the task allocation algorithm mentioned above to form a team for the task from the current active workers. Sometimes the allocation will fail because the number of active workers is insufficient. At this time, the requester can wait for new active workers to join the system and 
then call the allocation function again, or call the withdraw function to retrieve his prepaid budget. If the assignment is successful, the assigned worker should go to the task location to complete the task.

\section{Task Quality Evaluation And Payment}

The process of task quality assessment has been described in the previous section. When the task is finished, the original participants of the task should provide some evidence materials for scoring. And the Oracle Contract will use the method mentioned above to score the task. After the scoring is completed, either the requester or the workers can call the Payment(.) function to pay the workers. It is worth noting that the Payment $(\cdot)$ function only accepts one parameter, which is the task. Payment(.) function will query to Oracle Contract to get the score of the task and reward the honest workers who participated in the scoring. In this way, we can ensure that the scoring process is honest and the crowdsourcing process is fair.

\subsection{System feasibility analysis}

In this section, we mainly discuss the feasibility of the system. It is expanded from two aspects, one is the transaction delay problem of Ethereum blockchain mentioned in the third section, and the other is the time complexity of the task allocation algorithm.

\subsubsection{Analysis of Ethereum transaction delay problem}

Ethereum's transaction time delay is mainly caused by the block generation time set by the Ethereum system. At present, the block generation time of Ethereum is about 13s. In other words, every 13 seconds, Ethereum will process a batch of pending transactions. In the most ideal case, a transaction will be processed after one block interval(13s). Counting the transmission delay of the transaction in the Ethereum network, we can assume that the transaction has a basic delay of two block intervals. For the current design of Ethereum, its TPS(transaction per Second) is between 15-25[22]. Compared with traditional centralized systems, this is not a very high value. (e.g. Visa, 6000TPS). Once the number of transactions exceeds the capacity of Ethereum, it is easy to generate transaction congestion. For the period of mid-2020, Ethereum's average transaction delay reached 44 minutes. Excluding some extreme cases, most of the time the average transaction delay of Ethereum is between 2-5 minutes[23].

Moreover, the congestion waiting time caused by too many transactions can be controlled. If the requester needs to publish a very urgent task, the requester can appropriately increase the gas price and priority fee. For miners, packaging this transaction can get more revenue, which can naturally attract miners from the Ethereum network to prioritize packaging your emergency transaction. Of course, this will increase the transaction fee that needs to be paid for the transaction.

In terms of current Ethereum transaction congestion and throughput, the transaction delay will not exceed 5 minutes. If the task issued by the requester has a time limit of 3 hours, and the delay of the Ethereum transaction takes up about $2 \%$ of the time, we believe that this time delay is acceptable.

For multi-skilled spatial crowdsourcing tasks such as repairing water pipes and holding parties, due the commuting time of workers, most spatial crowdsourcing 
tasks will have a time limit of more than 3 hours. However, for Uber and Didi, this kind of time-critical crowdsourcing task, the 5 min delay may require users to carefully consider whether it is acceptable. According to the official Ethereum, ETH2.0 may be launched by the end of 2022. At that time, the system throughput of Ethereum will be improved by several orders of magnitude, and the transaction delay problem will be completely solved.

\subsubsection{Analysis of Algorithm time complexity}

We use a greedy task allocation algorithm based on skill coverage rate. And form a team of workers from all workers based on the skill requirements and spacetime constraints of the multi-skill spatial crowdsourcing task. In the first step, the algorithm uses the getValidWorker method to find the set of workers that meet the conditions from all workers. Secondly, the algorithm uses the formTeam method to form a team from all the valid workers according to the greedy strategy.

In the above process, getValidWorker method needs to traverse the worker set to find workers who meet the conditions. The formTeam method also needs to traverse the set of workers found in the last step to form a team. In each traversal, some calculations need to be done, such as judging whether the skills meet the requirements, calculating the skill coverage, and the logic of team formation. We set the time complexity of each traversal to be $t$, and the size of the worker to be $n$. The algorithm needs to traverse the worker set twice, so we can conclude that the time complexity of the algorithm is $O(2 n * t)$. In general, the proposed algorithm has linear time complexity, and its gas consumption is compared in detail in the experiment section.

Our algorithm mainly considers the skill coverage rate of workers, which means that the number of workers we select for a team will be as small as possible. This not only reduces the requester's budget consumption but also increases the overall task allocation rate. A higher task allocation rate also means fewer algorithm execution times. From this point of view, our algorithm can also reduce consumption on the Ethereum blockchain. The detailed experimental results are shown in the next section.

\section{Results and discussion}

The System we have implemented is deployed on the Ethereum blockchain. The optimization of the task allocation algorithm in the system can achieve a higher success rate of task assignment.

To evaluate these goals, we deploy the system on a local private chain and compare the task allocation success rate with an intuitive method.

\subsection{Program setup}

We use both real and synthetic data to test our proposed task allocation algorithm. The location and time data come from the real data set. Then we use a random algorithm to construct the skills of the workers and the skill requirements for the tasks. The specific data parameters are shown in the table1.

The smart contracts are deployed on the local private Ethereum blockchain. The programming language we used is solidity[24]. The truffle framework and ganache-cli are used to deploy and experiment the smart contracts. 
Table 1 Dataset Parameters

\begin{tabular}{ll}
\hline Number of worker & {$[60,120]$} \\
Location( latitude, longitude) & $([102,105],[102,105])$ \\
Time & {$[1615910403,1615996772]$} \\
Skills & {$[1,10]$} \\
\hline
\end{tabular}

\subsection{Task Allocation Process}

The blockchain-based crowdsourcing system proposed in [19] uses a task allocation algorithm based on time priority. And [18] proposed an auction-based mechanism to assign workers to a task, only considering the workers' bids for the task. To evaluate the superiority of our method, we implemented a FIFO method as a comparative experiment. The FIFO method simulates the task assignment process of [19],[18]. It will confirm whether the skills of the workers meet the task requirements, and then assigns tasks to the workers in the order of the time they joined the system.

As shown in Fig.8, the ordinate represents the success rate of task assignment, and the abscissa represents the number of workers in the system. We synthesized 3 sets of 25 task data to represent tasks of different difficulties. In Fig.8.(a), each task requires 3 skills, representing the simple task. Fig.8.(b) and Fig.8.(c) respectively represent a medium and difficult task. We compared the success rate of task assignment with 60,90 , and 120 workers, and adjust the difficulty of the task for comparative experiments.

Obviously, as the number of workers increases, the success rate of task assignments is rising. The greedy algorithm we proposed can easily achieve a success rate of more than $90 \%$ when the number of workers is sufficient. At the same time, when the task is difficult and the number of workers is small, the Greedy algorithm is also significantly better than the FIFO algorithm.

We can conclude that the Greedy algorithm has a higher task allocation rateIf we can assume that there are about 90 active workers in the system, then a $90 \%$ task allocation rate can be easily achieved through the greedy algorithm proposed.

\subsection{Smart Contract Cost}

In this experiment, we verified the economic feasibility of the proposed system. To achieve decentralization, the blockchain-based crowdsourcing system uses the Ethereum blockchain platform to replace the centralized platform in traditional crowdsourcing. There are currently 2,722 nodes[20] that maintain Ethereum globally. To incentive these miner nodes, the Ethereum platform charges a certain gas fee for each operation that consumes computer resources. To compare with TSWCrowd[19], we use the gas price and ether price of Ethereum in July 2020. 1 gas is set to $5^{*} 10^{\wedge}-9$ Ether, at the same time 1 Ether $=\$ 233.02(2020.7 .6)$. Table 2 shows the gas consumption in Ethereum.

Compared with TSWCrowd, the cost of our system is slightly higher. In the TSWCrowd, one round of task allocation costs $\$ 3.29$. In our system, the task allocation costs $\$ 5.9$, but a higher task allocation rate can ensure that the allocation is completed in one round of execution. In TSWCrowd, the number of rounds of algorithm execution may be higher, which will cause additional costs. 


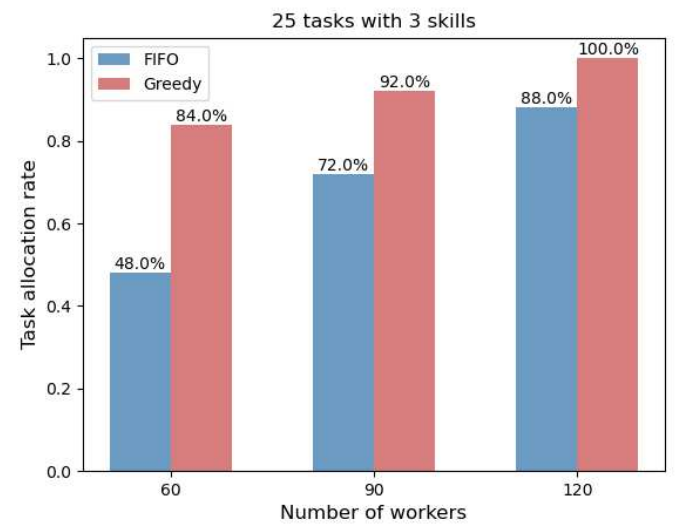

(a) simple



(b) middle

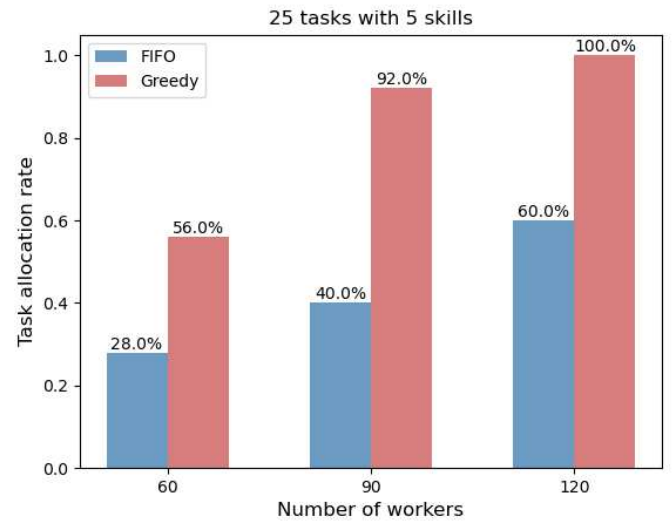

(c) hard

Figure 8 Comparison of the Success Rate of Task Assignment

The centralized platform Mechanical Turk (MTurk)[2] needs to pay at least a 20\% service fee to the platform to manage the crowdsourcing process. Therefore, the cost of our system to complete a crowdsourcing process is greatly reduced. 
Table 2 Gas Consumption of Main Function

\begin{tabular}{llll}
\hline Operation & gas cost & ether cost & USD cost \\
\hline Greedy Task Allocation & 5072974 & 0.02536 & 5.909 \\
FIFO Task Allocation & 2893366 & 0.01446 & 3.369 \\
UserRegister & 193025 & 0.000965 & 0.224 \\
PostTask & 276352 & 0.00138 & 0.321 \\
\hline
\end{tabular}

In conclusion, our system has good economic feasibility, which decentralizes the crowdsourcing process and significantly reduces the additional consumption of the crowdsourcing process.

\section{Conclusions}

The paper uses decentralized blockchain to replace the centralized crowdsourcing platform, thereby realizing a decentralized crowdsourcing system. In addition, a crowdsourcing task allocation algorithm is proposed, which realizes support for multi-skilled crowdsourcing tasks while ensuring a higher task allocation rate. Compared with traditional crowdsourcing, our system has two main advantages:1) The task allocation process is transparent and fair. 2) There is no centralized platform management crowdsourcing process, which can protect the privacy of users. The system uses the feature of the blockchain to realize the immutability of transactions and permanent storage, and the use of smart contract technology to ensure that the execution of the task allocation algorithm is fair and transparent. Moreover, the blockchain is an anonymous platform, which protects the privacy of users. Compared with the TSWCrowd blockchain-based crowdsourcing model, our system has a higher task allocation rate under the same conditions. While improving the completion rate of crowdsourcing tasks, it also reduces the additional cost of repeated calls to the allocation algorithm. And our system supports multi-skilled crowdsourcing tasks, which is more suitable for real spatial crowdsourcing application scenarios.

In future work, we will focus on optimizing the task allocation algorithms and user privacy protection. In the current method, each crowdsourcing task costs nearly $\$ 6.5$, which is still too expensive for some small and micro crowdsourcing tasks. Although the blockchain platform is anonymous, there is still the risk of exposure[25] on the chain, which means that the user's private information can be analyzed through a clustering attack. We want to find a way to execute the transparent and fair task allocation algorithm on-chain while protecting users' privacy. This can make blockchain crowdsourcing truly significant advantages compared with traditional crowdsourcing.

\footnotetext{
Acknowledgements

We sincerely thank the Reviewers and the Editor for their valuable suggestions.

Funding

This work is supported in part by by the Key R\&D Program of Zhejiang (2022C03087).

Abbreviations

GPS:Global Positioning System; WST:worker selects task; TSW:task selects worker; EVM:Ethereum Virtual Machine; DApps: decentralized blockchain applications; R-SMB: Repeated-Single- Minded Bidder; FIFO: first in first out; WMC:Worker Manager Contract; TMC:Task Manager Contract; TPS: transaction per second; ETH: ethereum.
} 
Availability of data and materials

Not applicable.

Ethics approval and consent to participate

Not applicable.

Competing interests

The authors declare that they have no competing interests.

Consent for publication

The authors declare that they agree with the consent for publication.

Authors' contributions

WX proposed the new idea of the paper and participated in the outage performance analysis. HD performed the simulations and drafted the paper. $\mathrm{JH}, \mathrm{DL}$ and $\mathrm{YC}$ conceived of the study, and participated in its design and coordination and helped to draft the manuscript. All authors have read and approved the final manuscript.

Author details

${ }^{1}$ Department of Cardiac Surgery, The Children's Hospital, Zhejiang University School of Medicine, National Clinical Research Center for Child Health, Hangzhou, China. ${ }^{2}$ School of Computer Science and Technology, Hangzhou Dianzi University, Hangzhou, China. ${ }^{3}$ School of Information and Electronic Engineering, Zhejiang University of Science and Technology, Hangzhou, China

\section{References}

1. Cheng, P., Lian, X., Chen, L., Han, J., Zhao, J.: Task assignment on multi-skill oriented spatial crowdsourcing IEEE Transactions on Knowledge and Data Engineering 28(8), 2201-2215 (2016). doi:10.1109/TKDE.2016.2550041

2. Amazon Mechanical Turk. https://www.mturk.com/. Accessed 2021

3. Uber. https://www.uber.com/. Accessed 2021

4. TaskRabbit. https://www.taskrabbit.com/. Accessed 2021

5. Li, M., Weng, J., Yang, A., Lu, W., Zhang, Y., Hou, L., Liu, J.-N., Xiang, Y., Deng, R.H.: Crowdbc: A blockchain-based decentralized framework for crowdsourcing. IEEE Transactions on Parallel and Distributed Systems 30(6), 1251-1266 (2019). doi:10.1109/TPDS.2018.2881735

6. Zhang, X., Xue, G., Yu, R., Yang, D., Tang, J.: Keep your promise: Mechanism design against free-riding and false-reporting in crowdsourcing. IEEE Internet of Things Journal 2(6), 562-572 (2015). doi:10.1109/JIOT.2015.2441031

7. Lu, Y., Tang, Q., Wang, G.: Zebralancer: Private and anonymous crowdsourcing system atop open blockchain. In: 2018 IEEE 38th International Conference on Distributed Computing Systems (ICDCS), pp. $853-865$ (2018) doi:10.1109/ICDCS.2018.00087

8. Liu, K., Chen, W., Zhang, Z.: Blockchain-empowered decentralized framework for secure and efficient software crowdsourcing. In: 2020 IEEE World Congress on Services (SERVICES), pp. 128-133 (2020). doi:10.1109/SERVICES48979.2020.00039

9. Lu, Y., Tang, Q., Wang, G.: On enabling machine learning tasks atop public blockchains: A crowdsourcing approach. In: 2018 IEEE International Conference on Data Mining Workshops (ICDMW), pp. 81-88 (2018). doi:10.1109/ICDMW.2018.00019

10. Kazemi, L., Shahabi, C.: Geocrowd: Enabling query answering with spatial crowdsourcing. In: Proceedings of the 20th International Conference on Advances in Geographic Information Systems. SIGSPATIAL '12, pp. 189-198. Association for Computing Machinery, New York, NY, USA (2012). doi:10.1145/2424321.2424346. https://doi.org/10.1145/2424321.2424346

11. Hu, A., Gu, Y.: Mobile crowdsensing tasks allocation for mult-parameter bids. In: 2017 IEEE 3rd Information Technology and Mechatronics Engineering Conference (ITOEC), pp. 489-493 (2017). doi:10.1109/ITOEC.2017.8122344

12. Qiao, R., Yan, S., Shen, B.: A reinforcement learning solution to cold-start problem in software crowdsourcing recommendations. In: 2018 IEEE International Conference on Progress in Informatics and Computing (PIC), pp. 8-14 (2018). doi:10.1109/PIC.2018.8706279

13. Tong, Y., She, J., Ding, B., Chen, L., Wo, T., Xu, K.: Online minimum matching in real-time spatial data: Experiments and analysis 9(12) (2016). doi:10.14778/2994509.2994523

14. Song, T., Xu, K., Li, J., Li, Y., Tong, Y.: Multi-skill aware task assignment in real-time spatial crowdsourcing. Geolnformatica 24(1), 153-173 (2020). doi:10.1007/s10707-019-00351-4

15. smart-contracts. https://www.ibm.com/topics/smart-contracts. Accessed 2021

16. Wang, S., Ouyang, L., Yuan, Y., Ni, X., Han, X., Wang, F.-Y.: Blockchain-enabled smart contracts: Architecture, applications, and future trends. IEEE Transactions on Systems, Man, and Cybernetics: Systems 49(11), 2266-2277 (2019). doi:10.1109/TSMC.2019.2895123

17. Xu, X., Pautasso, C., Zhu, L., Gramoli, V., Ponomarev, A., Tran, A.B., Chen, S.: The blockchain as a software connector. In: 2016 13th Working IEEE/IFIP Conference on Software Architecture (WICSA), pp. 182-191 (2016). doi:10.1109/WICSA.2016.21

18. Kadadha, M., Mizouni, R., Singh, S., Otrok, H., Ouali, A.: Abcrowd an auction mechanism on blockchain for spatial crowdsourcing. IEEE Access 8, 12745-12757 (2020). doi:10.1109/ACCESS.2020.2965897

19. Gao, L., Cheng, T., Gao, L.: Tswcrowd: A decentralized task-select-worker framework on blockchain for spatial crowdsourcing. IEEE Access 8, 220682-220691 (2020). doi:10.1109/ACCESS.2020.3043040

20. ethernodes. https://www. ethernodes.org/. Accessed 2021

21. BlockchainOracle. https://en.wikipedia.org/wiki/Blockchain_oracle. Accessed 2021

22. TPS. https://cryptotps.com/tps-capacity-bitcoin-ethereum-ripple-xrp-tron-eos/. Accessed 2021 
23. GasStation. https://ethgasstation.info/. Accessed 2021

24. Solidity. https://solidity.readthedocs.io/en/latest/. Accessed 2021

25. Meiklejohn, S., Pomarole, M., Jordan, G., Levchenko, K., McCoy, D., Voelker, G.M., Savage, S.: A fistful of bitcoins: Characterizing payments among men with no names. Commun. ACM 59(4), 86-93 (2016). doi:10.1145/2896384 